\title{
PROJECTIVE COVERS AND MINIMAL FREE RESOLUTIONS
}

\author{
MARK A. GODDARD \\ Department of Mathematical Sciences \\ The University of Akron \\ Akron, Ohio 44.325-4002 \\ (Received September 9, 1994)
}

ABSTRACT. Using a generalization of the definition of the projective cover of a module, a special type of surjective free resolution, known as the projective cover of a complex, may be defined. The projective cover is shown to be a direct summand of every surjective free resolution and to be the direct sum of the minimal free resolution and an exact complex. Necessary and sufficient conditions for the projective cover and minimal free resolution to be identical are discussed.

KEY WORDS AND PHRASES. Projective cover, free resolution, quasi-isomorphism.

1992 AMS SUBJECT CLASSIFICATION CODES. 16D40, 18G05,18G10.

\section{INTRODUCTION.}

Let $R$ be a commutative ring with identity. A complex $\mathbf{C}$ of $R$-modules is a sequence of $R$-module homomorphisms

$$
\ldots \rightarrow C_{n} \stackrel{\delta_{n}}{\rightarrow} C_{n-1} \rightarrow \ldots \rightarrow C_{1} \stackrel{\delta_{1}}{\rightarrow} C_{0} \rightarrow \ldots
$$

satisfying $\delta_{\imath-1} \circ \delta_{\imath}=0$ for all $\imath \in Z$. The maps $\delta_{i}$ are called the boundary maps of $\mathbf{C}$. In this paper, we will assume that all complexes are bounded above, i.e. $C_{\imath}=0$ for all $\imath<0$. If $\operatorname{ker} \delta_{\imath-1}=$ im $\delta_{\imath}$ for all $\imath \in Z$ then $\mathbf{C}$ is called an exact complex.

A map of complexes $\mathbf{C} \stackrel{\phi}{\rightarrow} \mathrm{D}$ is a sequence of $R$-module homomorphisms $C_{\imath} \stackrel{\phi_{2}}{\rightarrow} D_{2}$ which commutes with the boundary maps of the complexes $C$ and $D$. If each map $\phi_{2}$ is surjective, we will say that $\phi$ is surjective and if each induced map $H_{2}(\mathbf{C}) \stackrel{\phi_{i}^{*}}{\rightarrow} H_{2}(\mathbf{D})$ is an isomorphism, we will say that $\phi$ is a quasi- isomorphism. Generalizing the definition of the projective cover of a module, we define the projective cover of a complex $\mathbf{C}$ to be a complex $\mathbf{P}$ of projective modules and a map of complexes $\mathbf{P} \stackrel{\epsilon}{\rightarrow} \mathbf{C}$ with the following two properties:

1. For any complex $\mathbf{Q}$ of projective modules, and any map of complexes $\mathbf{Q} \stackrel{\phi}{\rightarrow} \mathbf{C}$, the diagram

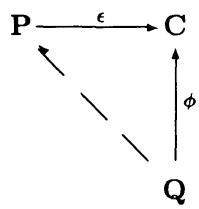


(an be completed by a map of complexes. (If $\mathbf{P} \stackrel{\epsilon}{\rightarrow} \mathbf{C}$ satisfies this condituon alone, then the map is called a projective precover.)

2. The diagram

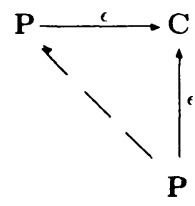

can only be completed by maps of complexes which are automorphisms of $P_{2}$ in each degree.

It can be shown (see Goddard|1]) that every bounded above complex of finitely generated modules over a Noetherian local ring has a projective cover and that the projective cover is a unique surjective quasi-isomorphism. The proofs of these facts depend upon two key lemmas which we shall find useful later in this paper as well. The first lemma, which is proved by Roberts[2] (pp. 42-44.), is helpful in proving that the projective cover is a surjective map of complexes.

LEMMA 1. If $\mathbf{C}$ is a bounded above complex of finitely generated modules over a Noetherian local ring then there exists a map of complexes $\mathbf{F} \stackrel{\phi}{\rightarrow} \mathbf{C}$ such that

1. Each $\operatorname{map} F_{2} \stackrel{\phi_{2}}{\rightarrow} C_{2}$ is surjective.

2. $F_{2}$ is a free module for each 2 .

3. $\phi$ is a quasi-isomorphism.

4. $\phi_{2}\left(Z_{2}(\mathbf{F})\right)=Z_{\imath}(\mathbf{C})$ for each $i$.

Our second lemma, which along with the first is used to prove that the projective cover is a quasi-isomorphism, has been proven by Goddard[1]. The proof is included here for completeness.

LEMMA 2. Let $\mathbf{C}$ be a bounded above complex. If $\mathbf{P} \stackrel{\boldsymbol{\epsilon}}{\rightarrow} \mathbf{C}$ is a surjective quasi-isomorphism then $\mathbf{P}$ is a projective precover of $\mathbf{C}$

PROOF. Let $\mathbf{Q}$ be a complex of projective modules and let $\mathbf{Q} \stackrel{\phi}{\rightarrow} \mathbf{C}$ be a map of complexes. We need to find a map $\mathbf{Q} \stackrel{h}{\rightarrow} \mathbf{P}$ so that $\epsilon \circ \mathbf{h}=\phi$. We let $h_{\imath}=0$ for $i<0$. For the $\imath=0$ case, we first need to note that for all $\imath \geq 0, \epsilon_{\imath}\left(Z_{\imath}(\mathbf{P})\right)=Z_{\imath}(\mathbf{C})=\left\{c \in C_{\imath} \mid \delta_{\imath}(c)=0\right\}$. In order to do so, let $c \in Z_{i}(\mathbf{C})$. Since $\epsilon$ is a quasi-isomorphism, there is a $p \in Z_{\imath}(\mathbf{P})$ such that $\epsilon_{\imath}(p)-c \in B_{\imath}(\mathbf{C})$. Since $\epsilon$ is surjective and commutes with the boundary maps, there exists $p^{\prime} \in P_{i+1}$ such that $\epsilon_{\imath}\left(b_{\imath+1}\left(p^{\prime}\right)\right)=\delta_{\imath+1}\left(\epsilon_{\imath+1}\left(p^{\prime}\right)\right)=\epsilon_{\imath}(p)-c$. Thus $c=\epsilon_{\imath}\left(p-b_{\imath+1}\left(p^{\prime}\right)\right) \in \epsilon_{\imath}\left(Z_{\imath}(\mathbf{P})\right)$.

Since $\epsilon_{0}\left(Z_{0}(\mathbf{P})\right)=Z_{0}(\mathbf{C})$ and $Q_{0}$ is projective, the diagram

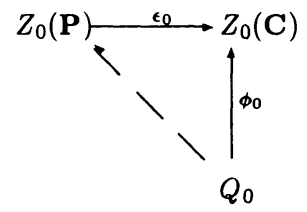

can be completed by a map $h_{0}$. We now proceed inductively to construct $\mathbf{h}$. Assume that we have constructed $h_{j}$ such that $\epsilon_{j} \circ h_{j}=\phi_{j}$ for all $j \leq i$ and $\mathbf{h}$ commutes with the boundary maps. We now wish to construct $h_{\imath+1}$. 


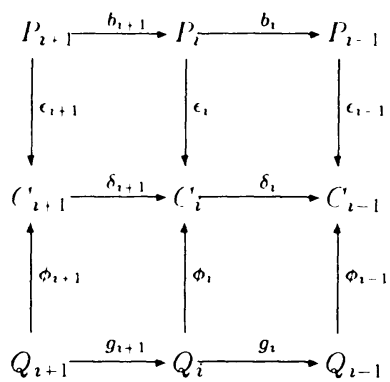

Since $\epsilon_{\imath} \circ h_{\imath} \circ g_{\imath+1}(q) \in B_{\imath}(\mathrm{C})$ for all $q \in Q_{\imath+1}$ and $\epsilon$ is a quasi-isomorphism, we have $h_{\imath} \circ g_{\imath+1}(q) \in$ $B_{\imath}(\mathbf{P})$ for all $q \in Q_{\imath+1}$. Thus the diagram

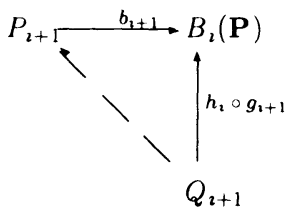

can be completed by a map $h^{\prime}$ since $Q_{\imath+1}$ is projective. For $q \in Q_{\imath+1}$, we have $\left(\epsilon_{\imath+1} \circ h^{\prime}-\phi_{\imath+1}\right)(q) \in$ $Z_{\imath+1}(\mathrm{C})$ so the diagram

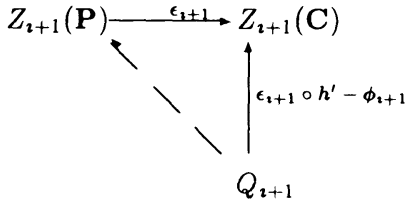

can be completed by a map $h^{\prime \prime}$ since the module $Q_{\imath+1}$ is projective and the map $Z_{\imath+1}(\mathbf{P}) \stackrel{\epsilon_{2+1}}{\rightarrow} Z_{\imath+1}(\mathbf{C})$ is surjective. If we let $h_{2+1}=h^{\prime}-h^{\prime \prime}$ then it follows easily that $\mathbf{h}$ commutes with the boundary and $\epsilon_{2+1} \circ h_{2+1}=\phi_{2+1}$.

Since the existence of projective covers and the hypotheses of lemma 1 both place the same requirements upon our complex $\mathbf{C}$, we shall assume hereafter that $\mathbf{C}$ refers to a bounded above complex of finitely generated modules over a Noetherian local ring. Under these conditions, the projective cover $\mathbf{P}$ like the complex $\mathbf{F}$ constructed in lemma 1, turns out to be a complex of free modules. Thus we define a free resolution of a complex $\mathbf{C}$ to be a quasi-isomorphism $\mathbf{F} \stackrel{\phi}{\rightarrow} \mathbf{C}$ from a complex of free modules into $\mathbf{C}$.

The free resolution of a complex $\mathbf{C}$ is not unique. The projective cover of $\mathbf{C}$ is one example of a surjective free resolution. Another unique type of free resolution is given below:

DEFINITION. A minimal free resolution of a complex $\mathbf{C}$ of $R$-modules is a free resolution $\mathbf{F} \stackrel{\phi}{\rightarrow} \mathbf{C}$ such that each boundary map $F_{\imath} \stackrel{f_{\imath}}{\rightarrow} F_{\imath-1}$ is defined by a matrix with entries in the maximal ideal $m$ of the local ring $R$.

Roberts[2] shows that every complex $\mathbf{C}$ has a unique minimal free resolution and that every free resolution is a direct sum of a minimal free resolution and an exact sequence. Later in this paper we will investigate the relationship between the projective cover and the minimal free resolution in detail. 


\section{SURJECTIVE FREE RESOLUTIONS}

According to lemma 2, cvery surjective free resolution of a complex $\mathrm{C}$ must be a projective precover of $\mathrm{C}$. The projective cover ttself is a unique example of a surjective free resolution but there may be other surjective free resolutions as well. For example, if $R=Z / 4$ and $\mathrm{C}$ is the bounded above complex

$$
\mathrm{C} \quad \rightarrow 0 \rightarrow Z / 4 \stackrel{\times 2}{\rightarrow} Z / 4 \rightarrow 0 \rightarrow \ldots
$$

then the projective cover of $\mathbf{C}$ is identical to $\mathrm{C}$ itself but if we utilize the construction used by Roberts $|2|$ to prove lemma 1 , we obtain a different surjective free resolution:

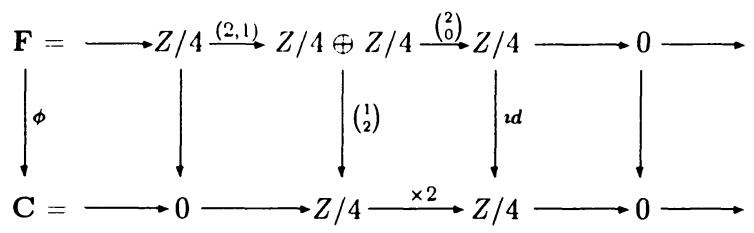

Notice in this case that the projective cover of $\mathrm{C}$ is identical to the minimal free resolution of $\mathrm{C}$. We will see in the next section how the structure of the complex $\mathbf{C}$ forces this to be true.

While the projective cover and surjective free resolution are not identical, they are very closely related. The exact nature of this relationship is seen in the following theorem:

THEOREM 1. The projective cover of a complex $\mathbf{C}$ is a direct summand of any surjective free resolution of the complex.

PROOF. Let $\mathbf{P} \stackrel{\epsilon}{\rightarrow} \mathbf{C}$ be the projective cover of $\mathbf{C}$ and let $\mathbf{F} \stackrel{\phi}{\rightarrow} \mathbf{C}$ be a surjective free resolution of $\mathbf{C}$. Since $\mathbf{P}$ is the projective cover of $\mathbf{C}$, the diagram

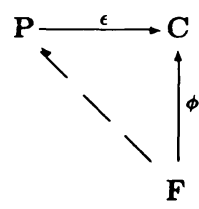

can be completed by a map of complexes $\mathbf{F} \stackrel{h}{\rightarrow} \mathbf{P}$. Also, since $\mathbf{F}$ is a projective precover of $\mathbf{C}$ (by lemma 2), the diagram

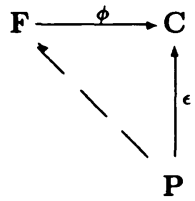

can be completed by a map of complexes $\mathbf{P} \stackrel{k}{\rightarrow} \mathbf{F}$. Thus the map $\mathbf{P} \stackrel{\text { hok }}{\rightarrow} \mathbf{P}$ completes the diagram

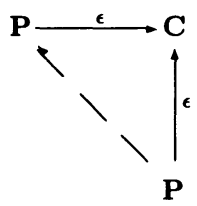

Since $\mathbf{P} \stackrel{\epsilon}{\rightarrow} \mathbf{C}$ is a projective cover, the map of complexes $h \circ k$ must be an automorphism of $\mathbf{P}$. Therefore, $h$ must be a surjection and we have the short exact sequence of complexes 


$$
0 \rightarrow \mathbf{K} \stackrel{\stackrel{\prime}{\rightarrow}}{\mathbf{H}} \stackrel{h}{\rightarrow} \mathbf{P} \rightarrow 0
$$

To see that $\mathbf{P}$ is a direct summand of $\mathbf{F}$, we simply need to show that there exists a map of complexes $\mathbf{P} \stackrel{g}{\rightarrow} \mathbf{F}$ such that $h \circ g=\imath d \mathbf{P} \quad$ At first glance, this may seem obrious sunce $\mathbf{P}$ is a complex of projective modules but it is not difficult to find an example of a complex of projective modules which does not form a projective complex (see Goddard|1|).

To obtain the map $\mathbf{g}$, we observe that both $\epsilon$ and $\phi$ must be quasi isomorphisms, so $\mathbf{h}$ is a quasi-1somorphism as well. Since $\mathbf{h}$ is also surjective, by lemma 2 , we know that $\mathbf{h}$ is a projective precover so the diagram

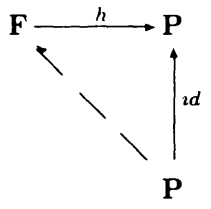

can be completed by the desired map of complexes $\mathbf{g}$.

3. MINIMAL FREE RESOLUTIONS

Just as the projective cover is a direct summand of the surjective free resolution, it is easy to see that the projective cover is a direct sum of the minimal free resolution and an exact complex. Although both the projective cover and the minimal free resolution are unique examples of free resolutions, they are not necessarily identical. For example, if $R=Z / 4$, the complex

$$
\mathbf{C}=\ldots \rightarrow 0 \rightarrow Z / 4 \stackrel{2 d}{\rightarrow} Z / 4 \rightarrow 0 \rightarrow \ldots
$$

is equal to its projective cover (The map $\mathbf{P} \stackrel{\epsilon}{\rightarrow} \mathbf{C}$ is simply the identity map) but is not equal to its minimal free resolution. In fact, the minimal free resolution of $\mathbf{C}$ is equal to the zero complex.

This example and the example of the previous section suggest that the relationship between the projective cover and the minimal free resolution depends upon the boundary maps of $\mathbf{C}$ and the maximal ideal $m$ of the ring $R$. Our next theorem will begin to formalize this relationship but we begin with a crucial lemma.

LEMMA 3. If $P \stackrel{\epsilon}{\rightarrow} M$ is the projective cover of the finitely generated $R$-module $M$ where $R$ is a local ring with maximal ideal $m, x \in P$, and $\epsilon(x) \in m M$ then $x \in m P$.

PROOF. The result follows easily from the construction of the projective cover (see Rot$\operatorname{man}[3]$, pp 136.).

THEOREM 2. If $\mathbf{C}$ is a complex of modules over a local ring $R$ with maximal ideal $m$, and $\mathbf{C}$ has boundary maps $\delta_{i}$ such that $\delta_{\imath+1}\left(C_{\imath+1}\right) \subseteq m \operatorname{ker}\left(\delta_{\imath}\right)$ for all $\imath$, then the projective cover $\mathbf{P} \stackrel{\epsilon}{\rightarrow} \mathbf{C}$ is equal to the minimal free resolution of $\mathbf{C}$.

PROOF. Let $b_{\imath}$ be the boundary map of the complex $\mathbf{P}$. To prove that $\mathbf{P}$ is the minimal free resolution, we must verify that $b_{\imath+1}\left(P_{\imath+1}\right) \subseteq m P_{\imath}$ for all $i \geq 0$. First, if $p \in P_{1}$ then

$$
\epsilon_{0}\left(b_{1}(p)\right)=\delta_{1}\left(\epsilon_{1}(p)\right) \in m C_{0}
$$

In the construction of the projective cover of a complex (see Goddard[1]), the map $P_{0} \stackrel{\epsilon_{0}}{\rightarrow} C_{0}$ is the (module) projective cover of $C_{0}$. Thus by the previous lemma, we see that $b_{1}(p) \in m P_{0}$.

Now suppose $x \in P_{\imath+1}$ for some $\imath \geq 1$. Once again invoking the construction of the projective cover 


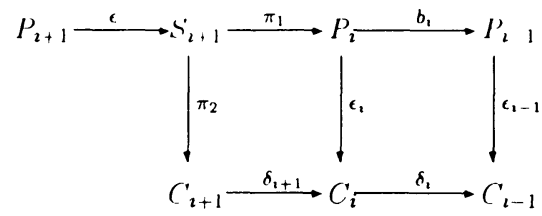

we recall that $P_{\imath+1}$ is the projective cover of the pullback module $S_{\imath+1}=\left\{(p, c) \in \operatorname{ker}\left(b_{\imath}\right) \oplus\right.$ $\left.C_{\imath+1} \mid \epsilon_{\imath}(p)=\delta_{\imath+1}(c)\right\}$ where $b_{\imath+1}=\pi_{1} \circ \epsilon$ and $\epsilon_{\imath+1}=\pi_{2} \circ \epsilon$. If $\epsilon(x)=(p, c) \in S_{\imath+1}$ then we need to show that $b_{\imath+1}(x)=p \in m P_{\imath}$.

Since $\delta_{2+1}(c) \in m \operatorname{ker}\left(\delta_{2}\right)$ by hypothesis, we know

$$
\delta_{\imath+1}(c)=r_{1} c_{1}++r_{n} c_{n}
$$

for some $r_{1}, r_{2}, \ldots, r_{n} \in m$ and $c_{1}, c_{2}, \ldots, c_{n} \in \operatorname{ker}\left(\delta_{\imath}\right)$. Since $P_{\imath}$ is the projective cover of $S_{\imath}=$ $\left\{\left(p^{\prime}, c^{\prime}\right) \in \operatorname{ker}\left(b_{\imath-1}\right) \oplus C_{2} \mid \epsilon_{\imath-1}\left(p^{\prime}\right)=\delta_{\imath}\left(c^{\prime}\right)\right\},\left(0, c_{\jmath}\right) \in S_{\imath}$ for $1 \leq \jmath \leq n$, and the projective cover is surjective, we can find elements $p_{1}, \quad, p_{n} \in \operatorname{ker}\left(b_{\imath}\right)$ such that $\epsilon_{2}\left(p_{\jmath}\right)=c_{\jmath}$ for $1 \leq j \leq n$. It follows that

$$
\epsilon_{2}\left(r_{1} p_{1}+\ldots+r_{n} p_{n}-p\right)=0
$$

and

$$
b_{2}\left(r_{1} p_{1}+\ldots+r_{n} p_{n}-p\right)=0
$$

so the image of $r_{1} p_{1}+\ldots+r_{n} p_{n}-p$ in $S_{\imath}$ is $(0,0) \in m S_{2}$. Again invoking the previous lemma, we have $r_{1} p_{1}+\ldots r_{n} p_{n}-p \in m P_{2}$ so $p \in m P_{2}$ as well.

One might wonder if we could weaken the hypotheses of our theorem slightly: If $\delta_{\imath+1}\left(C_{\imath+1}\right) \subseteq$ $m C_{\imath}$ for all $\imath$, is the projective cover always equal to the minimal free resolution? As we shall see shortly in an example, the answer is no, but first we observe that while this slightly stronger conjecture is false, its converse is true.

THEOREM 3. Let $\mathbf{C}$ be a complex of modules over a local ring $R$ with maximal ideal $m$ and boundary maps $\delta_{2}$. If the projective cover of $\mathrm{C}$ is equal to the minimal free resolution of $\mathrm{C}$ then $\delta_{\imath+1}\left(C_{\imath+1}\right) \subseteq m C_{\imath}$ for all $i$.

PROOF. Let $c \in C_{\imath+1}$ and let $\mathbf{P} \stackrel{\epsilon}{\rightarrow} \mathbf{C}$ be the projective cover of $\mathbf{C}$. Since the projective cover is surjective, there exists $p \in P_{i+1}$ such that $\epsilon_{i+1}(p)=c$. Since $\mathbf{P} \stackrel{\epsilon}{\rightarrow} \mathbf{C}$ is also the minimal free resolution of $\mathbf{C}, b_{i+1}(p) \in m P_{i}$ and $\epsilon_{\boldsymbol{\imath}}\left(b_{\imath+1}(p)\right) \in m C_{\boldsymbol{\imath}}$. Since $\epsilon$ commutes with the boundary maps, we have $\delta_{2+1}(c) \in m C_{2}$.

At this point we have the following three statements which can be made about a complex $\mathbf{C}$ :

1. $\delta_{\imath+1}\left(C_{i+1}\right) \subseteq m \operatorname{ker}\left(\delta_{\imath}\right)$

2. The projective cover of $\mathbf{C}$ is identical to the minimal free resolution of $\mathbf{C}$

3. $\delta_{\imath+1}\left(C_{i+1}\right) \subseteq m C_{\imath}$

We have seen that $(1) \Rightarrow(2) \Rightarrow(3)$ but in the following two examples, we will illustrate that statement (2) is equivalent to neither statement (1) nor statement (3).

First if we let $R=Z / 4$ with maximal ideal (2) and consider the complex

$$
\mathrm{C}=\ldots \stackrel{\times 2}{\rightarrow} Z / 4 \stackrel{\times 2}{\rightarrow} Z / 4 \stackrel{\times 2}{\rightarrow} Z / 4 \rightarrow 0 \rightarrow \ldots
$$


we have the projectwe cover and the mummal free resolution of $\mathrm{C}$ bothe equal to $\mathrm{C}$ itself where $\mathbf{P} \stackrel{\rightarrow}{\rightarrow}$ is the identitv map The complex $\mathbf{C}$ does not satisfv statement. (1) however so statements (1) and (2) are not equivalent

Next, if we let $R \quad Z / X$ with maximal ideal (2) and let.

$$
\mathrm{C}=\quad \rightarrow 0 \rightarrow Z / 8 \stackrel{\times 2}{\rightarrow} Z / 8 \stackrel{\times 4}{\rightarrow}(2) \rightarrow 0 \rightarrow
$$

which is a complex satisfying statement (3), we see that the projectuve cover $\mathbf{P} \stackrel{\epsilon}{\rightarrow} \mathbf{C}$ is given by:

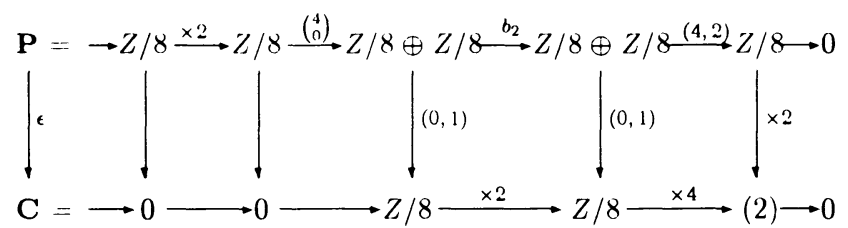

where the boundary map $b_{2}$ given by the matrix

$$
\left(\begin{array}{ll}
2 & 1 \\
0 & 2
\end{array}\right)
$$

contains an entry not $m$ the maximal ideal $m$. Thus $\mathbf{P} \stackrel{\epsilon}{\rightarrow} \mathbf{C}$ is not the minimal free resolution of $\mathbf{C}$ and statements (2) and (3) are not equivalent.

In order to give necessary and sufficient conditions for the projective cover and minimal free resolution to be identical, we must define a new subcomplex $\mathbf{D}$ of $\mathbf{C}$ such that statement (2) holds if and only if $\delta_{\imath+1}\left(C_{\imath+1}\right) \subseteq D_{\imath}$ for all $\imath$. From our discussion up to this point, we certainly need

$$
m k e r\left(\delta_{\imath}\right) \subseteq D_{\imath} \subseteq m C_{\imath}
$$

for all $i$. With this in mind, given the complex $\mathbf{C}$ with projective cover $\mathbf{P}$, we define the submodule $D_{\imath}$ of $C_{\imath}$ to be the set of elements $c \in C_{\imath}$ such that there exists elements $r_{1}, \ldots, r_{k}$ in the maximal ideal $m$, elements $c_{1}, \ldots, c_{k} \in C_{\imath}$ and elements $p_{1}, \ldots, p_{k} \in \operatorname{ker}\left(b_{\imath-1}\right)$ satisfying the following conditions:

1. $c=r_{1} c_{1}+\ldots+r_{k} c_{k}$

2. $\epsilon_{\imath-1}\left(p_{\jmath}\right)=\delta_{\imath}\left(c_{\jmath}\right)$ for $1 \leq j \leq k$

3. $r_{1} p_{1}+\ldots+r_{k} p_{k}=0$

It is easy to see with $D_{\imath}$ defined in this manner, that $\mathrm{D}$ is a subcomplex of $\mathrm{C}$ and that each $D_{\imath}$ satisfies the subset inequality given above.

THEOREM 4. The projective cover $\mathbf{P} \stackrel{\epsilon}{\rightarrow} \mathbf{C}$ of a complex $\mathbf{C}$ is equal to the minimal free resolution of $\mathbf{C}$ if and only if $\delta_{\imath+1}\left(C_{\imath+1}\right) \subseteq D_{\imath}$ for all $\imath \geq 0$.

PROOF. First, let us assume that $\mathbf{P} \stackrel{\epsilon}{\rightarrow} \mathbf{C}$ is the minimal free resolution of $\mathbf{C}$. In other words, if $b_{i}$ is the boundary map of $\mathbf{P}$ then $b_{\imath+1}\left(P_{\imath+1}\right) \subseteq m P_{\imath}$ for all $\imath$.

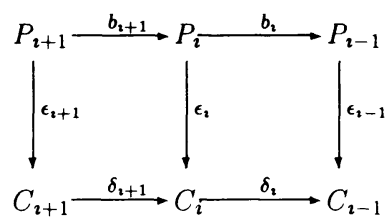

If $c \in C_{\imath+1}$, since $\mathbf{P} \stackrel{\epsilon}{\rightarrow} \mathbf{C}$ is surjective, there exists $p \in P_{\imath+1}$ such that $\epsilon_{\imath+1}(p)=c$. Since $b_{\imath+1}(p) \in m P_{\imath}$, we have 


$$
b_{1,1}(p) \quad r_{1} p_{1}^{\prime} 1+r_{k} p_{k}^{\prime}
$$

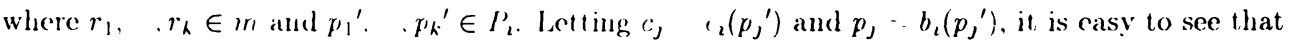
$\delta_{i+1}(c) \in D_{1}$.

Secondly; let us assume that $\delta_{\imath, 1}\left(\left(^{\prime} i+1\right) \subseteq D_{l}\right.$. We need to argue that if $x \in P_{\imath+1}$ then $b_{\imath+1}(x) \in m P_{\imath}$. If $c \quad \epsilon_{-1+1}(x)$ then we can find $c_{1} ., c_{k} \in\left({ }^{\prime}, p_{1}, \ldots, p_{k} \in \operatorname{ker}\left(b_{\imath-1}\right)\right.$ and $r_{1}, ., r_{k} \in m$ such that

1. $\delta_{2+1}(c)=r_{1} c_{1}+\quad+r_{h} c_{k}$

2. $\epsilon_{\imath-1}\left(p_{\jmath}\right)=\delta_{\imath}\left(c_{\jmath}\right)$ for $1 \leq \jmath \leq k$

3. $r_{1} p_{1}+\ldots+r_{k} p_{k}=0$

If we define $S_{2}=\left\{(p, c) \in \operatorname{ker}\left(b_{2-1}\right) \oplus C_{2} \mid \delta_{2}(c)=\epsilon_{2-1}(p)\right\}$ as in the proof of theorem 2 , then we have $\left(p_{j}, c_{\jmath}\right) \in S_{2}$ for $1 \leq \jmath \leq k$. Since the projective cover $P_{2} \stackrel{\epsilon}{\rightarrow} S_{2}$ is surjective, there exist elements $p_{1}{ }^{\prime}, \quad, p_{k}{ }^{\prime} \in P_{2}$ such that $\epsilon_{2}\left(p_{\jmath}{ }^{\prime}\right)=c_{\jmath}$ and $b_{\imath}\left(p_{\jmath}{ }^{\prime}\right)=p_{j}$ for $1 \leq j \leq k$.

If we let $p=b_{2+1}(x)$ then

$$
\epsilon_{\imath}\left(p-r_{1} p_{1}{ }^{\prime}-\cdot-r_{k} \cdot p_{k}{ }^{\prime}\right)=0
$$

and

$$
b_{z}\left(p-r_{1} p_{1}{ }^{\prime}-\ldots-r_{k} p_{k}{ }^{\prime}\right)=0
$$

so $p-r_{1} p_{1}{ }^{\prime}-\ldots-r_{k} p_{k}{ }^{\prime} \in \operatorname{ker}(\epsilon)$. Thus $\epsilon\left(p-r_{1} p_{1}{ }^{\prime}-\ldots-r_{k} p_{k}{ }^{\prime}\right) \in m S_{z}$ so by lemma 3 , it follows that $p \in m P_{2}$ and our proof is complete.

We close with an additional observation about the interrelationship between the projective cover and free resolutions. We recall that the surjective free resolution, being a projective precover, is almost equal to the projective cover. The minimal free resolution can be regarded as equally close to the projective cover for while the surjective free resolution always satisfies the first defining condition of a projective cover, it can be shown that the minimal free resolution always satisfies the second defining condition. The projective cover is in a sense squeezed in between, as it alone satisfies both conditions.

\section{REFERENCES}

1. GODDARD, M.A., Projective covers and quasi-isomorphisms, Rings, Extensions and Cohomology, (1994), 93-105.

2. ROBERTS, P., Homological Invarients of Modules over Commutative Rings, Les Presses de l'Université de Montreal, 1980.

3. ROTMAN, J., An Introduction to Homological Algebra, Academic Press, New York, 1979.

4. ENOCHS, E., Injective and flat covers, envelopes and resolvents, Israel Journal of Mathematics $\underline{3}, \underline{39}$ (1981), 189-209.

5. SILVER, L., Noncommutative localizations and applications, J. Algebra, 7 (1967), 44-75.

6. ROBERTS, P., Some remarks on the homological algebra of multiple complexes, Journal of Pure and Applied Algebra, 43 (1986), 99-110.

7. LAZARD, D., Autour de la platitude, Bull. Soc Math, $\underline{97}$ (1968), 82-128.

8. KAPLANSKY, I., Projective modules, Annals of Mathematics, $\underline{2}, \underline{68}$ (1958), 372-377. 


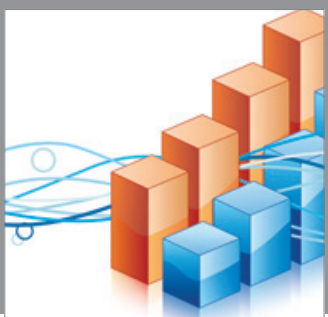

Advances in

Operations Research

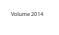

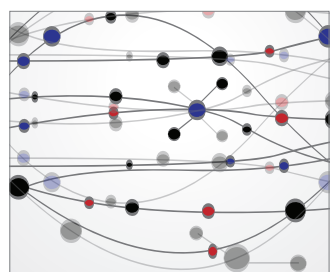

\section{The Scientific} World Journal
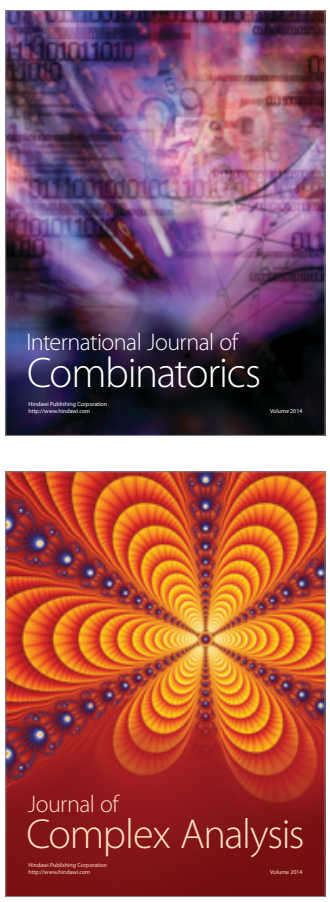

International Journal of

Mathematics and

Mathematical

Sciences
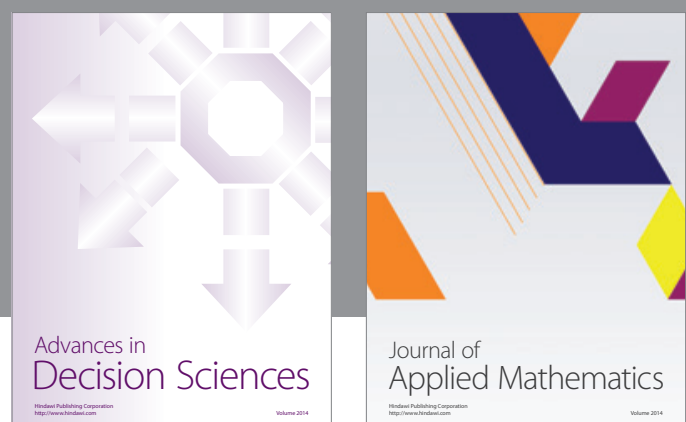

Journal of

Applied Mathematics
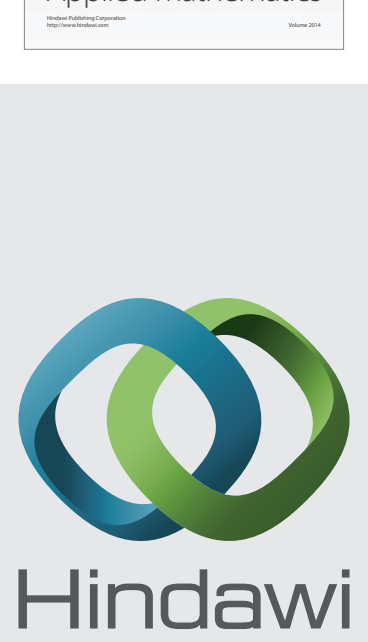

Submit your manuscripts at http://www.hindawi.com
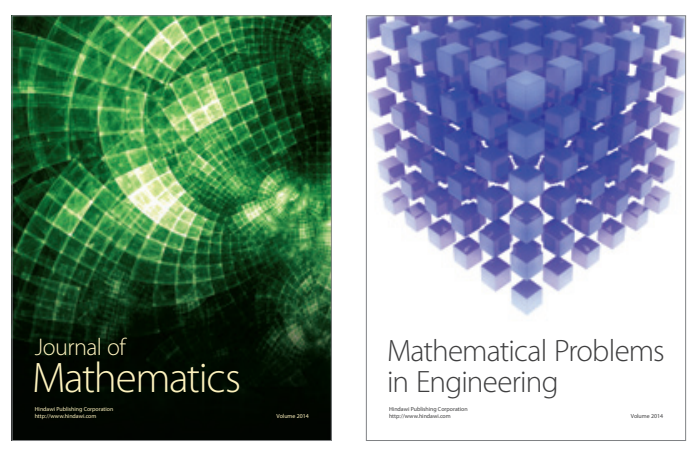

Mathematical Problems in Engineering
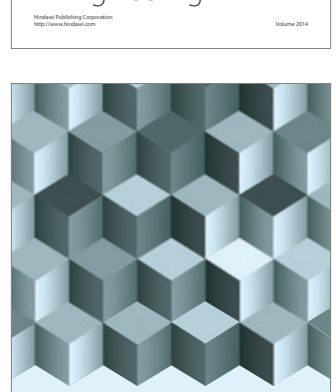

Journal of

Function Spaces
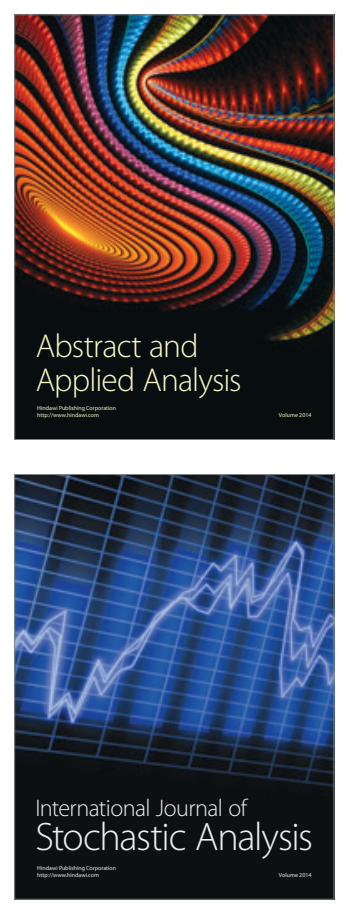

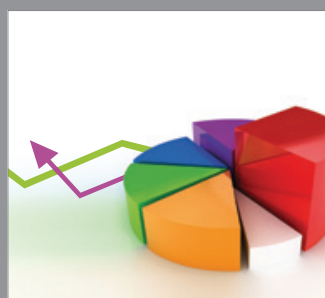

ournal of

Probability and Statistics

Promensencen
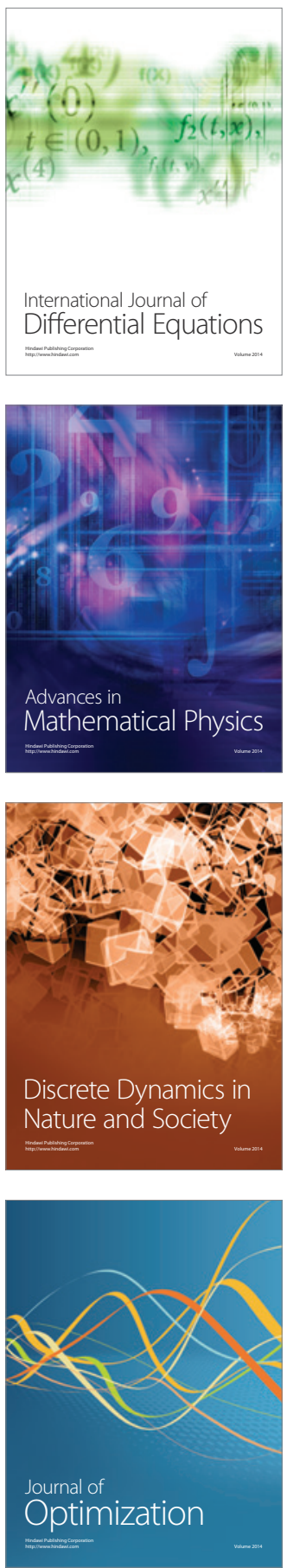